\title{
AVALIAÇÃO DA EXPOSIÇÃO OCUPACIONAL A MATERIAL BIOLÓGICO EM SERVIÇOS DE SAÚDE
}

\author{
Marília Duarte Valim¹, Maria Helena Palucci Marziale ${ }^{2}$
}

\footnotetext{
${ }^{1}$ Mestranda da Escola de Enfermagem de Ribeirão Preto (EERP) da Universidade de São Paulo (USP). São Paulo, Brasil. E-mail: duartevalim@usp.br

${ }^{2}$ Doutora em Ciências da Saúde. Professor Titular da Escola de Enfermagem de Ribeirão Preto da Universidade de São Paulo (EERP/USP). São Paulo, Brasil. E-mail: marziale@eerp.usp.br
}

\begin{abstract}
RESUMO: Acidente de trabalho com material biológico é agravo de notificação compulsória por associar-se ao risco de contaminação aos vírus HIV, HBV e HCV. Objetivou-se identificar a ocorrência e caracteristicas destes acidentes em instituições de saúde de um município do interior de São Paulo. Estudo descritivo, transversal, por meio de consulta ao Sistema de Informação de Agravos de Notificação, arquivados no Centro de Referência de Saúde do Trabalhador Regional de São João da Boa Vista, biênio 2008 -2009. Realizado estatística descritiva e apresentadas as proporções dos 85 acidentes registrados, com idades entre 19 e 57 anos; $85,9 \%$ sexo feminino; 73,4\% equipe de enfermagem; 8,2\% estudantes de enfermagem; 7,1\% médicos e 7,0\% trabalhadores da limpeza. Descarte de perfurocortantes em locais impróprios foi responsável por $18,8 \%$ dos acidentes de trabalho; em $80 \%$ houve contato com sangue e em $20 \%$ destes utilizou-se quimioprofilaxia. Há necessidade de melhoria da qualidade dos registros e investigação de possível subnotificação. DESCRITORES: Acidentes de trabalho. Exposição a agentes biológicos. Enfermagem.
\end{abstract}

\section{EVALUATING OCCUPATIONAL EXPOSURE TO BIOLOGICAL MATERIAL IN HEALTH SERVICES}

\begin{abstract}
Occupational accidents from exposure to biological material require mandatory notification due to potential contamination risks with HIV, HBV, and HCV. This study aimed to identify the occurrence and characteristics of occupational accidents in health care institutions located in the countryside of São Paulo, Brazil. This is a descriptive, cross-sectional study. Data was collected through consulting the registries of Information System for Notified Disease Exposures, filed in the São João da Boa Vista Regional Reference Center for Workers' Health database for 2008-2009. Descriptive statistics and frequencies of 85 occupational accidents registered were reported; aged 19 to 57 years, $85.9 \%$ were female; $73.4 \%$ nursing staff, $8.2 \%$ nursing students, $7.1 \%$ physicians, and $7.0 \%$ were cleaning personnel. Disposing sharp objects in inappropriate places accounted for $18.8 \%$ of occupational accidents; $80 \%$ had contact with blood, and $20 \%$ of them used chemoprophylaxis. It is necessary to improve the quality of records, on-the-job training, and investigation of possible under-reporting of occupational accidents.
\end{abstract}

DESCRIPTORS: Accidents, occupational. Exposure to biological agents. Nursing.

\section{EVALUACIÓN DE LA EXPOSICIÓN OCUPACIONAL A MATERIAL BIOLÓGICO EN SERVICIOS DE SALUD}

\begin{abstract}
RESUMEN: Los accidentes de trabajo con material biológico son de informe obligatorio por estar asociados con el riesgo de contaminación con los virus VIH, VHV y VHC. El objetivo del estudio fue identificar la incidencia y las características de los accidentes de trabajo en instituciones de salud, en un municipio del interior de São Paulo. Es un estudio descriptivo, transversal, con consulta al Sistema de Información de Notificaciones de Accidentes en el Centro Regional de Referencia de Salud de los Trabajadores, en São João da Boa Vista, SP, de 2008-2009. El estudio se realizó por estadística descriptiva y frecuencias de los 85 accidentes registrados; con edades entre 19 y 57 años; el 85,9\% mujeres; el 73,4\% personal de enfermería, el 8,2\% estudiantes de enfermería, el 7,1\% médicos, y el 7,0\% trabajadores de limpieza. El desecho de objetos punzantes en lugares inadecuados representó el 18,8\% de los accidentes en el trabajo, en el $80 \%$ había contacto con sangre, y el $20 \%$ utilizó la quimioprofilaxis. Hay necesidad de mejorar la calidad de los registros y la investigación de posible subregistro.
\end{abstract}

DESCRIPTORES: Acidentes de trabajo. Exposición a agentes biológicos. Enfermería. 


\section{INTRODUÇÃO}

A Lei Orgânica da Saúde n. ${ }^{\circ} 8080$, de 19/09/1990, regulamenta o Sistema Único de Saúde (SUS) brasileiro, e as ações em saúde do trabalhador, definidas, como um conjunto de atividades que se destinam, através das ações de vigilância epidemiológica e sanitária, à promoção e proteção da saúde dos trabalhadores, assim como visa à recuperação e reabilitação da saúde dos trabalhadores submetidos aos riscos e agravos advindos das condições de trabalho. ${ }^{1}$

A assistência à saúde do trabalhador deve se desenvolver integrada às ações de vigilância epidemiológica e sanitária, para que a dinâmica do processo saúde/doença decorrente do trabalho possa adquirir contornos mais definidos. As informações reunidas, analisadas, interpretadas e divulgadas como resultado de uma atuação integrada entre assistência e vigilância, certamente fará com que o papel atribuído ao sistema de vigilância, que é o de orientar as ações, seja cumprido. ${ }^{2}$

Neste contexto, os Acidentes de Trabalho (AT) são foco de atenção permanente das instituições e dos pesquisadores. De acordo com o Ministério da Saúde, Lei 8213, de 24 de julho de $1991^{3}$, o AT é aquele que acontece no exercício do trabalho e que traz como consequência, uma lesão corporal ou perturbação funcional, com perda ou redução da capacidade para o trabalho, de forma permanente ou temporária, ou até mesmo a morte.

O Ministério da Saúde (MS) dispõe sobre os procedimentos técnicos para a notificação compulsória de agravos à saúde do trabalhador, o que perfaz um total de 11 agravos, dentre os quais está regulamentado a notificação dos acidentes de trabalho com exposição a material biológico. A regulamentação da notificação destes agravos deve ser efetuada em ficha própria, padronizada pelo MS, no Sistema de Informação de Agravos de Notificação (SINAN-NET), e em redes sentinelas específica, a exemplo dos Centros de Referência de Saúde do Trabalhador (CEREST), para que as políticas de prevenção e controle possam ser executadas. ${ }^{4}$

Os CERESTs são unidades responsáveis por ações de prevenção, promoção, diagnóstico, tratamento, reabilitação e vigilância em saúde dos trabalhadores urbanos e rurais, independentemente do vínculo empregatício e do tipo de inserção no mercado de trabalho. ${ }^{5}$

A exposição ocupacional por material biológico é entendida como a possibilidade de contato com sangue e fluidos orgânicos no ambiente de trabalho e as formas de exposição incluem inoculação percutânea, por intermédio de agulhas ou objetos cortantes, e o contato direto com pele e/ ou mucosas. ${ }^{6}$

O Ministério do Trabalho e Emprego ${ }^{7}$ classifica os riscos biológicos como sendo aqueles que compreendem as exposições ocupacionais aos agentes biológicos, que são microorganismos geneticamente modificados ou não, as culturas de células, os parasitas, as toxinas e os príons. $\mathrm{O}$ MS, em conjunto com a Coordenação Nacional de Saúde do Trabalhador (COSAT), elaborou em 2006 um novo protocolo para atendimento aos profissionais expostos a material biológico, o qual segue um fluxograma de atendimento e notificação das exposições. ${ }^{8}$

Dentre os vários tipos de acidentes, os perfurocortantes são não só os mais frequentes, como também os mais graves, por possibilitarem o desenvolvimento de doenças letais para os trabalhadores. ${ }^{9}$

Estima-se que ocorram 385.000 casos de acidentes ao ano com material perfurocortante em trabalhadores de saúde nos hospitais americanos. ${ }^{10}$ Investigação de casos de infecção pelo vírus HIV, nesse grupo de trabalhadores, pelo Center for Disease Control and Prevention (CDC), identificou 57 casos de soroconversão e 140 possíveis casos de AIDS pós-exposição ocupacional, no período de 1981 a 2006.

A referida fonte ressalta que o acidente percutâneo foi responsável por 48 casos, seguido do muco-cutâneo (cinco casos). Em dois casos, houve associação dessas exposições e em dois casos aconteceu com material desconhecido. Os fluidos envolvidos foram sangue (49 casos), fluido com sangue visível (um caso), fluido inespecífico (quatro casos) e concentrado de vírus em laboratório (três casos).

Dentre as ocupações estudadas, a enfermagem foi a mais acometida, com 24 casos de soroconversão e 35 possíveis casos de infecção ocupacional pelo vírus HIV, respectivamente. ${ }^{11}$ Porém, deve-se ressaltar que o número de profissionais infectados ocupacionalmente no Brasil ainda é desconhecido.

A notificação como registro documental se faz importante para que as estimativas da ocorrência de acidentes biológicos sejam divulgadas assim como a letalidade das infecções, o que se faz considerar que os hospitais necessitam voltar maior atenção ao problema, direcionar medidas para a notificação dos acidentes, melhorar o encaminhamento dos trabalhadores acidentados aos 
serviços especializados e adotar medidas para a prevenção dos acidentes nos locais de trabalho. ${ }^{12}$

Os fatores de risco para aquisição do $\mathrm{HCV}$ após exposição ocupacional dependem do grau de contato com o sangue ou com instrumentos cortantes e da prevalência de anti-HCV entre os pacientes e o risco de transmissão está na média de 1,8\%.Em relação ao HBV, o risco pode variar de 6 a $30 \%$. Em relação ao HIV, o risco de transmissão ocupacional é cerca de 0,3 a $0,5 \%$ na exposição percutânea. O risco após exposição de mucosas, como os olhos, nariz e boca está estimado em $0,1 \%$. O risco de exposição da pele não íntegra ao sangue infectado pelo vírus está avaliado a ser menor que $0,1 \%{ }^{10}$

Frente à realidade, as instituições de saúde passaram a adotar medidas de segurança aos trabalhadores de saúde quanto à exposição a sangue e fluídos biológicos, quando da realização de suas atividades, denominada atualmente de precauções-padrão.

Embora muitos trabalhadores aceitem as normas de biossegurança, estas ainda não permeiam a prática diária com a mesma intensidade, fato resultante ao sentimento de invulnerabilidade dos trabalhadores. ${ }^{13}$

A redução dos ATs, com exposição a material biológico, requer educação em segurança e saúde e adesão às práticas seguras de trabalho, redução da utilização de procedimentos invasivos (tanto quanto possível), um ambiente de trabalho seguro e uma razão adequada de profissionais nas equipes de saúde pela proporção de pacientes assistidos. ${ }^{10}$

Desta forma, a análise epidemiológica dos acidentes com exposição a material biológico contribuirá para que as informações relacionadas a este tipo frequente de acidente possam ser identificadas e relatadas e para que políticas de prevenção e controle possam ser adotadas, tanto pelas instituições de saúde quanto pelos CERESTs.

O objetivo desse estudo foi identificar a ocorrência dos acidentes de trabalho com exposição a material biológico, em instituições de saúde vinculadas ao Centro de Referência de Saúde do Trabalhador, e as características destes acidentes.

\section{MÉTODOS}

Trata-se de um estudo descritivo, de corte transversal e com abordagem quantitativa dos dados.

Os critérios de inclusão adotados para a realização do presente estudo englobou os pro- fissionais lotados em instituições de saúde de São João da Boa Vista, com registros de notificação de AT com material biológico ao CEREST Regional deste município. A coleta de dados se deu no mês de março de 2010, e por esse motivo, optou-se pela consulta ao biênio 2008-2009.

Assim, a população de estudo foi composta pelas fichas de notificação dos Acidentes de Trabalho, com exposição a material biológico, no Sistema de Agravos de Notificação (SINAN-NET), emitidas nos anos de 2008 e 2009, envolvendo trabalhadores lotados em instituições de saúde. As instituições notificadoras foram assim categorizadas:

- Unidade A: um Hospital filantrópico de 224 leitos.

- Unidade B: 17 instituições vinculadas à Prefeitura Municipal (oito Unidades Básicas de Saúde (UBS), cinco Programas de Saúde da Família - PSF, um Ambulatório de Atenção Especializada (SAE), dois Laboratórios de Análises Clínicas (LAC) e um Pronto Socorro (PS)).

- Unidade C: um hospital particular vinculado à Cooperativa.

- Unidade D: uma instituição de saúde especializada em doenças renais.

Foi realizada consulta documental aos registros do CEREST e das fichas de notificação do SINAN NET envolvendo trabalhadores de saúde que sofreram acidentes ocupacionais com exposição a material biológico.

As características dos acidentes registrados foram obtidas por meios das seguintes variáveis: categoria profissional, tipo de exposição, material orgânico envolvido, circunstância do acidente, agente causal, uso de Equipamento de Proteção Individual (EPI) no momento do acidente, situação vacinal do trabalhador com relação à Hepatite $B$ e condutas pós exposição ocupacional. Ademais, foram calculados os Coeficientes de Riscos (CRs) para cada categoria profissional exposta ao risco de acidente de trabalho com material biológico, sendo este o resultado do total de acidentados de cada instituição, pelo total de trabalhadores expostos.

As informações foram coletadas em fevereiro e março de 2010, por uma das autoras da investigação. As informações foram registradas (considerando dupla digitação, validação e checagem da consistência das informações) e codificadas em planilha eletrônica, em forma de banco de dados Excel for Windows, transferidas e analisadas pelo programa Epi-Info versão 3.5.1. 
O projeto foi aprovado pelo Comitê de Ética em Pesquisa da Escola de Enfermagem de Ribeirão Preto/USP, conforme Resolução 196/96, do Conselho Nacional de Saúde. ${ }^{14}$ Para a realização do estudo, foi solicitada a autorização à coordenadoria do CEREST de São João da Boa Vista. O projeto foi autorizado sob protocolo 1128/2010.

\section{RESULTADOS E DISCUSSÃO}

No biênio 2008-2009 foram registrados no SINAN NET, 85 ATs entre trabalhadores das instituições de saúde estudadas. Os resultados, segundo as variáveis estudadas, estão apresentados a seguir.

Tabela 1 - Caracterização dos trabalhadores vítimas de acidente de trabalho com exposição a material biológico no biênio 2008-2009, lotados em instituições de saúde de um município do interior de São Paulo

\begin{tabular}{lrrrrrr}
\hline \multirow{2}{*}{ Variáveis } & \multicolumn{2}{c}{$\mathbf{2 0 0 8}(\mathbf{n}=\mathbf{4 7})$} & \multicolumn{2}{c}{$\mathbf{2 0 0 9}(\mathbf{n}=\mathbf{3 8})$} & \multicolumn{2}{c}{ Total $\mathbf{( n = 8 5 )}$} \\
\cline { 2 - 7 } Idade (anos) & $\mathbf{n}$ & $\mathbf{9}$ & $\mathbf{n}$ & $\mathbf{\%}$ & $\mathbf{n}$ & $\mathbf{\%}$ \\
$19 \mid-30$ & 20 & 42,5 & 16 & 42,1 & 36 & 42,4 \\
$30 \mid-40$ & 14 & 29,8 & 18 & 47,4 & 32 & 37,6 \\
$40 \mid-50$ & 10 & 21,3 & 4 & 10,5 & 14 & 16,5 \\
$\geq 50$ & 3 & 6,4 & - & - & 3 & 3,5 \\
Sexo & & & & & & \\
Feminino & 41 & 87,2 & 32 & 84,2 & 73 & 85,9 \\
Masculino & 6 & 12,8 & 6 & 15,8 & 12 & 14,1 \\
Categoria profissional & & & & & & \\
Auxiliar de enfermagem & 20 & 42,5 & 22 & 57,9 & 42 & 49,4 \\
Técnico de enfermagem & 6 & 12,8 & 6 & 15,8 & 12 & 14,1 \\
Enfermeiro & 3 & 6,4 & 5 & 13,2 & 9 & 9,4 \\
Médico & 5 & 10,6 & 1 & 2,6 & 6 & 7,1 \\
Trabalhadores de limpeza & 4 & 8,5 & - & - & 4 & 4,7 \\
Estudante de enfermagem & 6 & 12,8 & 1 & 2,6 & 7 & 8,2 \\
Outros & 3 & 6,4 & 3 & 7,9 & 6 & 7,1 \\
Instituição de saúde & & & & & & \\
Hospital filantrópico & 21 & 44,7 & 18 & 47,4 & 39 & 45,9 \\
Prefeitura municipal & 11 & 23,4 & 9 & 23,7 & 20 & 23,5 \\
Hospital particular & 10 & 21,3 & 9 & 23,7 & 19 & 22,4 \\
Instituição especializada em doenças renais & 5 & 10,6 & 2 & 5,2 & 7 & 8,2 \\
\hline
\end{tabular}

Com relação à faixa etária, a idade mínima dos expostos foi de 19, e a idade máxima, 57 anos. A média foi de 31 anos (Tabela 1). Em relação ao sexo, $85,9 \%$ dos acidentes corresponde ao sexo feminino, o que condiz com os achados na literatura. ${ }^{15-16}$ Os resultados desvelam que não se percebe grandes mudanças com relação ao perfil dos trabalhadores de enfermagem vítimas de AT com exposição biológica, o que reafirma as características da força de trabalho de enfermagem no Brasil, sendo esta predominantemente feminina, e com idade inferior a 40 anos. ${ }^{17}$

A maior parte dos acidentados corresponde à classe dos auxiliares de enfermagem $(49,4 \%)$, dos técnicos de enfermagem $(14,1 \%)$, dos enfermeiros $(9,4 \%)$, dos estudantes de enfermagem $(8,2 \%)$, médicos $(7,1 \%)$, e dos profissionais da limpeza $(7,0 \%)$, que corresponde aos faxineiros $(4,6 \%)$ e auxiliares de lavanderia (2,4\%) (Tabela 1). De acordo com a literatura, os técnicos e auxiliares de enfermagem são os profissionais mais propensos a acidentes com material biológico, por estarem em contato direto com o paciente, em maior parte do tempo, e devido à realização de atividades inerentes à profissão. $^{13}$

Os profissionais da equipe de enfermagem somam $72,9 \%$ dos acidentados, o que condiz com achados de outros estudos, ${ }^{18}$ cuja categoria 
representou um total de $73 \%$ dos acidentes registrados em um Hospital Universitário de São Paulo (Tabela 1).

Os estudantes de enfermagem foram a segunda categoria acometida por tais injúrias, que têm, nas unidades de saúde, a oferta de campos de estágio pelas instituições de ensino. $\mathrm{O}$ manuseio de perfurocortantes e a possibilidade de exposição mucocutânea por estudantes da área da saúde é frequente na atividade acadêmica, o que os expõe ao risco de adquirir infecção ocupacionais. ${ }^{19}$ Os médicos correspondem a 7,1\%, seguidos dos profissionais da limpeza $(7,0 \%)$, os quais merecem especial atenção, uma vez que se muitas vezes se deparam com materiais sem fonte conhecida, o que intensifica a gravidade da exposição.

Notificações a um CEREST de um município do Paraná evidenciou que os estagiários de enfermagem ocupavam a segunda posição entre os expostos, precedido pelos auxiliares de enfermagem e seguidos pelos técnicos de enfermagem e classe médica. ${ }^{20}$ Observou-se que $84,7 \%$ dos expostos acidentaram-se com materiais perfurocortantes, $\mathrm{o}$ que confere com achados na literatura. ${ }^{6,16-18}$
Evidenciou-se que $72,9 \%$ dos acidentados estavam utilizando luvas de procedimento e $68,2 \%$ utilizavam o avental. Destaca-se com relação ao uso do avental que a baixa adesão dos profissionais a esta precaução-padrão subestima a Norma Regulamentadora 32 (NR 32), que trata exclusivamente da Segurança e Saúde no Trabalho em Serviços de Saúde (Tabela 2).

A referida norma ressalta a importância do uso dos EPIs frente à realização de procedimentos com risco de exposição a sangue ou fluídos corpóreos, e enfatiza o uso de vestimentas de proteção à saúde dos trabalhadores, a exemplo dos aventais, que devem ser obrigatoriamente utilizados, devendo ser colocado no início e retirado quando do fim de suas atividades laborativas, em local exclusivo e adequado para este fim, a cargo da instituição empregadora. ${ }^{21}$

Os trabalhadores de saúde, muitas vezes desconhecem essas informações e acreditam que o uso do EPI não diminui a inoculação de sangue, o que tem sido uma barreira para adequada utilização de luvas principalmente na administração de medicações por via endovenosa. ${ }^{22}$

Tabela 2 - Distribuição dos acidentes de trabalho, segundo o uso de equipamento de segurança individual no momento do acidente ocorridos nas instituições de saúde de um município do interior de São Paulo, no biênio 2008-2009

\begin{tabular}{|c|c|c|c|c|c|c|c|c|c|c|c|c|c|c|c|c|c|c|c|}
\hline \multirow{3}{*}{ Variáveis } & \multicolumn{7}{|c|}{$2008(n=47)$} & \multicolumn{6}{|c|}{$2009(n=38)$} & \multicolumn{6}{|c|}{ Total $(n=85)$} \\
\hline & \multicolumn{2}{|c|}{ Sim } & \multicolumn{2}{|c|}{ Não } & \multicolumn{3}{|c|}{ Ignorado } & \multicolumn{2}{|c|}{ Sim } & \multicolumn{2}{|c|}{ Não } & \multicolumn{2}{|c|}{ Ignorado } & \multicolumn{2}{|c|}{ Sim } & \multicolumn{2}{|c|}{ Não } & \multicolumn{2}{|c|}{ Ignorado } \\
\hline & $\mathbf{n}$ & $\%$ & $\mathbf{n}$ & $\%$ & $\mathbf{n}$ & \% & $\%$ & $\mathbf{n}$ & $\%$ & $\mathbf{n}$ & $\%$ & $\mathbf{n}$ & $\%$ & $\mathbf{n}$ & $\%$ & $\mathbf{n}$ & $\%$ & $\mathbf{n}$ & \\
\hline $\begin{array}{l}\text { Uso de EPI } \\
\text { Luvas }\end{array}$ & 36 & 76,6 & 11 & 23,4 & & - & - & 26 & 68 & 12 & 31,6 & - & - & 62 & 72,9 & 23 & 27,1 & - & - \\
\hline Avental & 15 & 31,9 & 32 & 68,1 & & - & - & 9 & 24 & 26 & 68,4 & 3 & 8 & 24 & 28,2 & 58 & 68,2 & 3 & 4 \\
\hline Óculos & 4 & 8,5 & 43 & 91,5 & & - & - & 4 & 11 & 31 & 81,6 & 3 & 8 & 8 & 9,4 & 74 & 87,1 & 3 & 4 \\
\hline Máscara & 6 & 12,8 & 41 & 87,2 & & - & - & 5 & 13 & 30 & 79 & 3 & 8 & 11 & 12,9 & 71 & 83,5 & 3 & 4 \\
\hline Proteção facial & 2 & 4,2 & 45 & 95,7 & & - & - & - & - & 34 & 89,5 & 4 & 11 & 2 & 2,4 & 79 & 92,9 & 4 & 5 \\
\hline Boca & 2 & 4,2 & 45 & 95,7 & & - & - & 2 & 5,3 & 33 & 86,8 & 3 & 8 & 4 & 4,7 & 78 & 91,8 & 3 & 4 \\
\hline
\end{tabular}

* EPI - Equipamento de Proteção Individual

O sangue esteve presente em $80 \%$ das exposições; é o material biológico que tem os maiores títulos do vírus da Hepatite B e é o principal responsável pela transmissão ocupacional do vírus. ${ }^{20}$ Fluídos com sangue foram responsáveis por $4,7 \%$. O plasma sanguíneo foi relatado em dois acidentes, e o líquor, em apenas um relato de exposição. Outros materiais foram responsáveis por $4,7 \%$ e o material foi ignorado em $7,0 \%$ das notificações.
Quanto ao tipo de exposição sofrida pelos trabalhadores, em 2008 foram encontradas 41 exposições percutâneas, e em 2009 relatou-se 31 exposições, o que resultam em $84,7 \%$ dos casos notificados. Os ATs com exposição de mucosa envolveram nove trabalhadores no biênio estudado $(10,6 \%)$. O envolvimento de pele íntegra foi relatado em $31 \%$ dos acidentados e não houve relatos de exposição ocupacional de material biológico potencialmente contaminado à pele não íntegra. 
Tabela 3 - Distribuição dos acidentes de trabalho segundo as circunstâncias causadoras dos acidentes ocorridos nas instituições de um município do interior de São Paulo, no biênio 2008-2009

\begin{tabular}{|c|c|c|c|c|c|c|}
\hline \multirow{2}{*}{ Variáveis } & \multicolumn{2}{|c|}{$2008(n=47)$} & \multicolumn{2}{|c|}{$2009(n=38)$} & \multicolumn{2}{|c|}{ Total $(n=85)$} \\
\hline & $\mathrm{n}$ & $\%$ & $\mathbf{n}$ & $\%$ & $\mathbf{n}$ & $\%$ \\
\hline \multicolumn{7}{|l|}{ Circunstâncias do acidente } \\
\hline Administração de medicação endovenosa & 4 & 8,6 & 3 & 7,9 & 7 & 8,2 \\
\hline Administração de medicação intramuscular & 1 & 2,1 & 1 & 2,6 & 2 & 2,4 \\
\hline Administração de medicação subcutânea & - & - & - & - & - & - \\
\hline Administração de medicação intradérmica & - & - & - & - & - & - \\
\hline Punção venosa/arterial para coleta sangue & 2 & 4,2 & 2 & 5,3 & 4 & 4,7 \\
\hline Punção venosa/arterial não especificada & 2 & 4,2 & 2 & 5,3 & 4 & 4,7 \\
\hline Descarte perfurocortante em saco de lixo & 2 & 4,2 & 1 & 2,6 & 3 & 3,5 \\
\hline Descarte perfurocortante em outros locais & 10 & 21,3 & 6 & 15,8 & 16 & 18,8 \\
\hline Lavanderia & - & - & 2 & 5,3 & 2 & 2,4 \\
\hline Lavagem de material & - & - & 1 & 2,6 & 1 & 1,2 \\
\hline Manipulação de caixa com perfurocortante & 5 & 10,6 & 3 & 7,9 & 8 & 9,4 \\
\hline Procedimento cirúrgico & 3 & 6,4 & - & - & 3 & 3,5 \\
\hline Procedimento odontológico & - & - & 1 & 2,6 & 1 & 1,2 \\
\hline Procedimento laboratorial & 3 & 6,4 & - & - & 3 & 3,5 \\
\hline Dextro & 6 & 12,8 & 6 & 15,8 & 12 & 14,1 \\
\hline Reencape & 3 & 6,4 & - & 0 & 3 & 3,5 \\
\hline Outros & 6 & 12,8 & 10 & 26,3 & 16 & 18,8 \\
\hline Ignorado & - & - & - & - & - & - \\
\hline
\end{tabular}

Em 18,8\% dos acidentes a ocorrência foi por descarte de perfurocortante em locais inadequados (leito, bancada, chão) e, igualmente, em 18,8\% dos casos, não houve especificação do procedimento executado. Em sequência, 14,1\% dos acidentes se deram na realização da glicemia capilar e 9,4\%, durante a manipulação de caixas coletoras de materiais perfurocortantes. A ocorrência de acidentes por descarte inadequado, descarte em saco de lixo comum, manipulação da caixa coletora e encape ativo somam 35,2 \% dos acidentes, situações passíveis de prevenção, e o não preenchimento da atividade realizada $(18,8 \%)$ no momento do acidentes dificulta a investigação (Tabela 3).

A agulha foi a maior responsável pelo acidentes, com $74,1 \%$ das notificações, fato condizente com os achados na literatura nacional, ${ }^{18}$ e as com lúmen, foram causadoras de 72,9\% dos acidentes.

As lâminas/lancetas foram identificadas como agente causal em apenas 3,5\% dos registros, enquanto que as notificações de glicemia capilar/ dextro somaram $14,1 \%$ dos ATs. Frente ao exposto, há um indicativo de que este procedimento não esteja sendo realizado com lancetas, o que alerta para que ações educativas sejam direcionadas aos profissionais de saúde, a fim de encorajar o uso de lancetas quando da realização de glicemia capilar/ dextro, uma vez que a presença de sangue residual no interior das agulhas com lúmen intensifica a gravidade do acidente. ${ }^{10}$

A fonte era conhecida em $77,6 \%$ dos casos. Os testes sorológicos evidenciaram dois casos de positividade para o vírus HIV, um caso para a Hepatite B e três pacientes para o vírus da Hepatite $C$, o que condiz com a gravidade dos acidentes aos trabalhadores. Com relação às condutas tomadas no momento do acidente, tem-se que, no biênio estudado, 20\% dos profissionais acometidos por exposição a material biológico recorreram à quimioprofilaxia, o que totalizou 17 vítimas deste AT. $\mathrm{Na}$ grande maioria das indicações foi prescrito o esquema de antiretroviral AZT + 3TC (Zidovudina e Lamivudina). Em apenas um caso das exposições houve indicação de AZT + 3TC + Nelfinavir.

$O$ atendimento ao acidentado deve se realizar imediatamente, de preferência nas primeiras duas horas após o acidente. O trabalhador de saúde necessita ser monitorado e orientado no momento do acidente e durante todo o período de tratamento prescrito. Atenção especial deve ser dada à vítima, 
já que a exposição biológica o afeta psicológica e emocionalmente, devido à espera dos resultados dos testes sorológicos e a possível soroconversão. ${ }^{23}$

O esquema vacinal para Hepatite B apresentou-se completo em 99,8\%. Os testes sorológicos dos acidentados, no momento do acidente, revelaram que não havia positividade para os vírus do HIV e hepatites B e C, porém não houve preenchimento em $20 \%$ dos acidentes. A pesquisa pelo Anti-HBs verificou que $40 \%$ dos profissionais apresentam o anticorpo e consequente resposta vacinal; em $16,1 \%$ o acidentado era negativo para o Anti-HBs; $18,5 \%$ não realizaram o teste e houve $20 \%$ de não preenchimento do importante dado. Percebeu-se que, mesmo com a ausência da resposta vacinal de alguns trabalhadores, não se relatou a utilização de quimioprofilaxia para a hepatite $B$.

Após o esquema completo de vacinação, se faz necessária a avaliação da soroconversão para o anti-HBs em grupos de risco, imunocomprometidos, e para os profissionais de saúde, já que nem sempre a imunização é eficaz. ${ }^{24}$

Com relação aos testes sorológicos do paciente, fonte e as condutas pós-exposição, a exemplo da Hepatite $C$, não há no momento profilaxia existente. $\mathrm{O}$ tratamento preconizado para trabalhadores que se acidentaram com fonte $\mathrm{HCV}$ positiva consiste na realização de alguns exames, sendo que no momento do acidente deve-se realizar o teste sérico de alanina aminotransferase (ALT) e prosseguir ao monitoramento. ${ }^{8}$

Os CRs das instituições estudadas, utilizando-se como referência o total de profissionais nas categorias ocupacionais mais acometidas (auxiliares e técnicos de enfermagem, enfermeiros, médicos, trabalhadores de limpeza e estudantes de enfermagem), mostram que na Unidade $A$, no ano de 2008, de 98 auxiliares de enfermagem, 11 estiveram expostos $(C R=11,2)$. Notificaram-se cinco acidentes com estudantes de enfermagem em campo de estágio, vinculados à instituição superior de ensino, no município, que correspondiam a um total de $35(C R=14,3)$. Houve relato de apenas uma notificação envolvendo trabalhadores da limpeza e enfermeiro, respectivamente, e apenas três notificações de pessoal médico. Assim, do total de 296 trabalhadores, houve 21 expostos $(C R=7,0)$.

Em 2009, com 280 trabalhadores expostos, notificou-se 17 acidentes $(C R=6,0)$. Os auxiliares de enfermagem somaram dez acidentes $(C R=10,20)$ e apenas um caso envolvendo estudantes de enfermagem $(C R=2,9)$. Verificou-se que três enfermeiros sofreram exposição, de um total de 21 trabalhadores $(C R=14,3)$. Os técnicos de enfermagem e o pessoal médico relataram apenas um caso de exposição e não houve notificação de AT com exposição a material biológico envolvendo o pessoal da limpeza.

Na Unidade B em 2008, 11 acidentes foram notificados de 209 empregados $(C R=5,3)$. Seis auxiliares de enfermagem acidentaram-se dos 91 expostos $(C R=6,6)$. Não houve notificações com técnicos de enfermagem e enfermeiros no total das 17 instituições de saúde. Em 2009 notificaram-se apenas sete exposições em 217 trabalhadores $(C R=3,3)$. Ressalta-se a ocorrência de acidente com um auxiliar de laboratório e um dentista. Em 2009 foi relatado um total de seis exposições, sendo cinco destas envolvendo auxiliares de enfermagem.

Na Unidade C, dos 114 empregados em 2008, dez apresentaram notificações direcionadas ao SINAN $(C R=8,8)$. De 47 técnicos de enfermagem, quatro acidentaram-se $(\mathrm{CR}=8,5)$. Houve dois auxiliares de enfermagem acometidos por este AT de 41 expostos $(\mathrm{CR}=4,8)$ e um indivíduo de 15 profissionais da limpeza $(C R=6,7)$. Neste ano houve dois acidentados das categorias de auxiliar de laboratório e agente de faturamento. Em 2009 registraram-se apenas nove exposições de 131 empregados $(C R=6,9)$, sendo quatro auxiliares de enfermagem $(\mathrm{CR}=8,9)$, um enfermeiro $(\mathrm{CR}=7,7)$ um profissional da limpeza $(C R=14,3)$ e um auxiliar de lavanderia.

$\mathrm{Na}$ Unidade D, de 68 trabalhadores, cinco registros foram constatados no banco de dados coletado $(\mathrm{CR}=7,35)$. Dois técnicos de enfermagem estiveram expostos de um total de $32(\mathrm{CR}=6,3)$, um enfermeiro de um total de seis (CR=16,7) e um profissional da limpeza de oito contratados $(C R=12,5)$. Em 2009 apenas dois técnicos de enfermagem $(\mathrm{CR}=5,1)$ foram expostos com devida notificação.

O resultado da evolução do caso do profissional acidentado mostrou que a maioria dos acidentados $(48,2 \%)$ obteve alta devido ao paciente fonte ter os resultados das sorologias negativos. Em 22,4\% dos casos foi registrado alta sem conversão sorológica e não houve notificações de soroconversão. No entanto, deve-se ressaltar que em 3,5\% dos casos houve abandono por parte do acidentado e $25,9 \%$ das fichas de notificação não preencheram este importante dado (Tabela 4).

Frente ao exposto, deve-se exaltar a necessidade de acompanhamento do profissional acidentado pelo mínimo de seis meses, com a realização de testes sorológicos e nova reabertura de notificação no SINAN NET bem como a necessidade de correto preenchimento da notificação. 
Tabela 4 - Evolução do caso do profissional acidentado com exposição biológica ocorridos em instituições de saúde de um município do interior de São Paulo, no biênio 2008-2009

\begin{tabular}{lrrrrrr}
\hline \multirow{2}{*}{ Variáveis } & \multicolumn{2}{c}{$\mathbf{2 0 0 8}(\mathbf{n}=\mathbf{4 7})$} & \multicolumn{2}{c}{$\mathbf{2 0 0 9}(\mathbf{n}=\mathbf{3 8})$} & \multicolumn{2}{c}{ Total (n=85) } \\
\cline { 2 - 7 } & $\mathbf{n}$ & $\mathbf{0}$ & $\mathbf{n}$ & $\mathbf{\%}$ & $\mathbf{n}$ & $\mathbf{\%}$ \\
\hline Evolução do caso & & & & & & \\
Alta com conversão sorológica & - & - & - & - & - & - \\
Alta sem conversão sorológica & 13 & 27,6 & 6 & 15,8 & 19 & 22,4 \\
Alta paciente fonte negativo & 21 & 44,7 & 20 & 52,6 & 41 & 48,2 \\
Abandono & 3 & 6,4 & - & - & 3 & 3,5 \\
Óbito por acidente com material biológico & - & - & - & - & - & - \\
Óbito por outra causa & - & - & - & - & - & - \\
Ignorado & - & - & - & - & - & - \\
Não preenchido & 10 & 21,3 & 12 & 31,6 & 22 & 25,9 \\
\hline
\end{tabular}

Com relação à abertura da $\mathrm{CAT}$, mesmo com a notificação ao SUS, os acidentes ou doenças ocupacionais também deverão ser comunicados à Previdência Social, por meio desta ferramenta de notificação. ${ }^{25}$

As categorias profissionais com maior CR, conforme descrito anteriormente, foram os enfermeiros ( $C R=16,7$ e $C R=14,3)$, os estudantes de enfermagem $(\mathrm{CR}=14,3)$ e os profissionais da limpeza $(C R=14,3$ e 12,5), respectivamente.

Entre as quatro unidades de saúde, o hospital particular cooperado obteve a maior proporção de acidentados, em 2008 (CR=8,8). A instituição especializada em doenças renais aparece em segundo lugar $(C R=7,35)$ no ano de 2008, porém com um coeficiente de risco menor em $2009(\mathrm{CR}=2,9)$. Na sequência, tem-se o hospital filantrópico, no ano de $2008(C R=7,0)$, com ligeiro declínio em 2009 $(C R=6,0)$. As unidades de saúde ligadas à prefeitura municipal, com registros no CEREST, aparecem com a menor proporção de notificações no biênio ( $C R=6,6$ em 2008 e $C R=3,3$ em 2009).

As atividades de promoção em saúde no trabalho se intensificaram nas últimas décadas, com o objetivo de redução de acidentes com materiais biológicos, porém, devido à falta de conscientização dos trabalhadores e das gerências das instituições de saúde, muitas vezes a notificação deste tipo de acidente não é realizada, demonstrando números de ocorrência que não condizem com a realidade. ${ }^{17}$

Supõe-se que a Unidade B, em especial, esteja sofrendo subnotificação dos ATs, tendo em vista o grande número de instituições de saúde relacionadas e o grande número de procedimentos invasivos e procedimentos supostamente realizados, à exemplo do Pronto Socorro, o Ambulatório de
Especialidades Médicas e as UBSs, como instituições integrantes. Sabe-se que muitos profissionais desconhecem o fluxograma preconizado frente à exposição biológica ocupacional, relacionada à pouca importância dada pelo trabalhador ou desconhecimento e negligência por parte dos profissionais responsáveis.

\section{CONCLUSÕES}

Os acidentes de trabalho com exposição a material biológico é uma realidade preocupante nas instituições de saúde. As lacunas de preenchimento de informações importantes revelam a necessidade de treinamento dos responsáveis para os registros e dificultam a elaboração de estratégias preventivas.

Torna-se necessário conscientizar os trabalhadores da necessidade da adesão às precauções- padrão, uso de Equipamento de Proteção Individual (EPI) e das instituições cumprirem as exigências da Norma Regulamentadora - NR 32. Outros estudos devem ser realizados nestas instituições, com a finalidade de detectar se há subnotificação dos ATs. As instituições de saúde, os supervisores e os trabalhadores devem se conscientizar mutuamente para a adoção de estratégias preventivas e a melhoria da qualidade dos registros dos acidentes.

\section{REFERÊNCIAS}

1. Brasil. Lei 8080 de 19 de setembro de 1990: dispõe sobre as condições para promoção, proteção e recuperação da saúde, a organização e o funcionamento dos serviços correspondentes e dá outras providências. Brasília, 1990.

2. Ministério da Saúde (BR). Saúde do trabalhador: cadernos de atenção básica - n 5. Brasília (DF): MS; 2002. 
3. Brasil. Lei $n^{\circ} .8213$ de 24 de julho de 1991: dispõe sobre os planos de benefícios da Previdência Social e dá outras providências. Diário oficial da União, Brasília: Ministério da Saúde, 14 Jul 1991 [acesso 2009 Jul 12]. Disponível em: http://www81. dataprev.gov.br/sislex/paginas/42/1991/8213.htm

4. Minsitério da Saúde (BR). Portaria $n^{0} 777 /$ GM de 28 de abril de 2004: dispõe sobre os procedimentos técnicos para a notificação compulsória de agravos à saúde do trabalhador em redes de serviço sentinela específica, no Sistema Único de Saúde. Brasília (DF): MS; 2004 [acesso 2010 Fev 02]. Disponível em: http://dtr2001.saude.gov.br/sas/PORTARIAS/ Port2004/GM/GM-777.htm

5. Ministério da Saúde (BR). Portaria N o 2.437, de 7 de dezembro de 2005: dispõe sobre a ampliação e o fortalecimento da Rede Nacional de Atenção Integral à Saúde do Trabalhador no Sistema Único de Saúde e dá outras providências. Brasília (DF): MS; 2005.

6. Gomes GP, Pinho DL M, Rodrigues CM. Perfil dos acidentes de trabalho no hospital universitário de Brasília. Rev. Brasileira Enferm. 2007 Mai-Jun; 60(3):291-4.

7. Ministério do Trabalho e Emprego (BR). Riscos biológicos - guia técnico: os riscos biológicos no âmbito da NR 32. Brasília (DF): MTE; 2008.

8. Ministério da Saúde (BR). Secretaria de Atenção à Saúde. Departamento de Ações Programáticas Estratégicas. Exposição a materiais biológicos. Brasília (DF): Ministério da Saúde; 2006.

9. Felli VEA, Sarquis LMM. Acidentes de trabalho com instrumentos perfurocortantes entre trabalhadores de enfermagem. Rev. Esc. Enferm. USP. 2002 Set; 36(3):222-30.

10. Centers for disease control and prevention (CDC). Workbook for designing, implementing and evaluating a sharp injury prevention program [online]. 2008 [access 2009 Jun 05]. Available from: www.cdc.gov/sharpssafety.

11. Centers for disease control and prevention (CDC). Surveillance of occupationally acquired HIV/ AIDS in healthcare personnel, as of December 2006 [online]. Set 2007 [acesso 2010 Abr 04]. Disponível em: http://www.cdc.gov/ncidod/dhqp/pdf/bbp/ fact_sheet_clearance_revised_090507Dec2006.pdf

12. Ferreira MM, Marziale MPH, Nishimura KYN. Riscos de contaminação ocasionados por acidentes de trabalho com material perfurocortante entre trabalhadores de enfermagem. Rev Latino-am Enfermagem. 2004 Jan-Fev; 12(1):36-42.

13. Padilha MICS, Vieira M. O HIV e o trabalhador de enfermagem frente ao acidente com material perfurocortante. Rev Esc Enferm. USP. 2008 Dez; 42(4):804-10.
14. Ministério da Saúde (BR), Conselho Nacional de Saúde, Comissão Nacional de Ética em Pesquisa. Resolução n ${ }^{\circ} 196$ de 10 de outubro de 1996: diretrizes e normas regulamentadoras de pesquisa envolvendo seres humanos. Brasília (DF): MS; 1996.

15. Chiodi MB, Marziale MHP, Robazzi MLCC. Occupational accidents involving biological material among public health workers. Rev Latino-am Enfermagem. 2007 Jul-Ago; 15(4):632-8.

16. Castro DMB, Pinto ER, Coutinho GAG, Bugari LF, Jesus MSB. Acidente de Trabalho entre profissionais de enfermagem de um hospital público de Porto Velho, Rondônia - Brasil. Cad. Saúde Coletiva. 2008 Nov-Dez; 25(5):206-11.

17. Marziale MHP. Ocurrencia de accidentes de trabajo causados por material corto-puzante entre trabajadores de enfermería en hospitales de la región nordeste de São Paulo, Brasil. Ciencia y Enfermería. 2003 Jun; 9(1):21-30.

18. Balsamo AC, Felli VEA. Estudo sobre os acidentes de trabalho com exposição aos líquidos corporais humanos em trabalhadores da saúde de um hospital universitário. Rev. Latino-am Enfermagem. 2006 Mai-Jun; 14(3):343-56.

19. CIcolini G, Di Labio L, Lancia L. Prevalence of biological exposure among nursing students: an observational study. Prof Inferm. 2008 Oct-Dec; 61(4):217-22.

20. Spagnuolo RS, Baldo RCS, Guerrini I. A. Análise epidemiológica dos acidentes com material biológico registrados no Centro de Referência em Saúde do Trabalhador - Londrina-PR. Rev Bras Epidemiol. 2008 Jun; 11(2):315-23.

21. Ministério do Trabalho e Emprego (BR). Portaria n. 485 de 11 de novembro de 2005: dispõe sobre a Norma Regulamentadora NR-32 relativa a segurança e saúde no trabalho em serviços de saúde. Diário Oficial de União, Brasília, 16 Nov 2005.

22. Zapparoli, AS, Marziale MHP, Robazzi MLCC. Práctica segura del uso guantes em la uncion venosa por los trabaljadores de enfermeria. Cienc Enfermeria. 2006 Dez; 12(2):63-72.

23. Sailer GC, Marziale MHP. Vivência dos trabalhadores de enfermagem frente ao uso dos antiretrovirais após exposição ocupacional a material biológico. Texto Contexto Enferm. 2007 Jan-Fev; 16(1):55-62

24. Pinheiro J, Zeitoune RCG. Hepatite B: conhecimento e medidas de biossegurança e a saúde do trabalhador de enfermagem. Esc Anna Nery Rev Enferm. 2008 Jun; 12(2):258-61

25. Ribeiro, MCS, organizador. Enfermagem e Trabalho: fundamentos para a atenção à saúde dos trabalhadores. São Paulo (SP): Martinari; 2008. 